\title{
AVALIAÇÃO DA CONTAMINAÇÃO DE METAIS EM SEDIMENTOS SUPERFICIAIS DAS PRAIAS DO LAGO DE ITAIPU
}

\author{
Andressa Belo e Sueli Pércio Quináia* \\ Departamento de Química, Universidade Estadual do Centro-Oeste, Rua Camargo Varela de Sá, 3, 85040-080 Guarapuava - PR, Brasil \\ Adelmo Lowe Pletsch \\ Departamento de Ciências Biológicas e Tecnologia, Universidade Estadual do Norte do Paraná, BR 369, km 54, 86360-000 \\ Bandeirantes - PR, Brasil
}

Recebido em 17/4/09; aceito em 13/10/09; publicado na web em 10/3/10

\begin{abstract}
ASSESSMENT OF CONTAMINATION OF METALS IN SEDIMENT SURFACE OF THE BEACHES FROM THE ITAIPU LAKE. This work was performed to evaluate the distribution of metals $\mathrm{Al}, \mathrm{Cd}, \mathrm{Cr}, \mathrm{Cu}, \mathrm{Fe}, \mathrm{Mn}, \mathrm{Ni}, \mathrm{Pb}$ and $\mathrm{Zn}$ in the sediment surface in the Itaipu Lake-PR-Brazil. It was also performed to measurement the $\mathrm{pH}$, phosphorus, organic carbon and particle size. In accordance of international criteria of quality of sediment, the results indicate an anthropogenic collaborations since some metals reached an excessive values. The factor of contamination was also used to evaluate the levels of contamination. The levels of $\mathrm{Cu}$ and $\mathrm{Pb}$ indicate a moderate contamination, so that it's possible to do harm to the balance of the ecosystem studied.
\end{abstract}

Keywords: metals; sediment; Itaipu Lake.

\section{INTRODUÇÃO}

Os níveis de metais tóxicos no meio ambiente têm aumentado seriamente nas últimas décadas. O crescimento industrial desordenado gera como consequência uma grande liberação de compostos indesejáveis ao meio ambiente, causando danos às vidas animal e vegetal. Desta forma, apesar dos bens minerais contribuírem para o desenvolvimento industrial são, também, fontes poluidoras quando lançados em grande quantidade ao meio ambiente. ${ }^{1,2}$

$\mathrm{O}$ lançamento dos efluentes industriais e domésticos, o intemperismo e outros processos pedogênicos que geram os elementos-traço sobre a rocha matriz podem propiciar um perfil completamente diferente ao da dinâmica de sedimentação encontrada nos ambientes naturais não perturbados. Estas incorporações que ocorrem, geralmente, de maneira lenta induzem a considerar erroneamente que não trazem efeitos graves, no entanto, levam à poluição dos ecossistemas aquáticos ou terrestres e consequente contaminação por metais-traço. ${ }^{3}$

A partir desta incorporação, podemos apontar como grandes impactadores os compostos inorgânicos, especialmente íons metálicos, que podem desempenhar dupla função na fisiologia dos organismos: alguns são indispensáveis para a vida, enquanto que outros são tóxicos, podendo afetar a atividade das espécies levando à perda de funções vitais, deformidade de órgãos e, em alguns casos, à morte. ${ }^{4,5}$

Os fenômenos de acúmulo e de redisposição de espécies nos sedimentos qualificam-nos como de extrema importância em estudos de impacto ambiental, pois registram em caráter mais permanente os efeitos de contaminação. A determinação de metais-traço em sedimentos permite quantificar o estoque mobilizável de um determinado contaminante em um local específico e, assim, detectar o grau de contaminação a que a água e os organismos bentônicos estão sujeitos ao longo do tempo. ${ }^{2,6}$ Por esta razão, é necessário contar com ferramentas de avaliação e controle que permitam estimar o risco existente tanto para a saúde dos ecossistemas aquáticos, como para a saúde humana.

O nível de contaminação dos metais no sedimento pode ser avaliado a partir de um fator de contaminação (FC), obtido através da razão entre a concentração do elemento no sedimento superficial e

*e-mail: spquinaia@gmail.com a concentração do seu nível de base natural (NBN) ou background. Essa metodologia permite verificar em que ordem de grandeza o nível natural dos metais foi excedido, indicando o enriquecimento ou, caso contrário, o empobrecimento do elemento no sedimento. ${ }^{7}$

O Lago de Itaipu, região de estudo, caracteriza-se pelas praias artificiais criadas a partir da formação da Usina Hidrelétrica de Itaipu, uma das maiores represas do planeta, que possui uma forte relação com o seu entorno e com a inserção regional, tendo como objetivo primordial, enquanto complexo hidrelétrico, a geração de energia. Os fins secundários consistem nos usos múltiplos do reservatório: navegação, recreação e turismo, pesca e captação de água para fins industriais, urbanos e rurais. ${ }^{8}$

Este trabalho descreve uma avaliação da contaminação dos sedimentos aquáticos provenientes de praias artificiais do Lago de Itaipu, exploradas por milhares de banhistas e veranistas, para alguns elementos metálicos, com a indicação das áreas mais críticas em relação à contaminação. Parâmetros físico-químicos como $\mathrm{pH}$, fosfato total $\left(\mathrm{P}_{\mathrm{T}}\right)$, carbono orgânico total (COT) e análise granulométrica também foram medidos. Essa avaliação foi elaborada através de comparações com critérios internacionais de indicativos da qualidade dos sedimentos descritos na literatura.

\section{PARTE EXPERIMENTAL}

\section{Local da coleta e preparo das amostras}

As amostras de sedimento superficial $(2 \mathrm{~cm})$ foram coletadas em nove pontos distribuídos ao longo do Lago de Itaipu, PR nos meses de março e maio de 2008 (Figura 1S, material suplementar). A profundidade da coleta do sedimento superficial foi de, aproximadamente, $1 \mathrm{~m}$ de coluna d'água, exceto para o ponto 3 , sendo próximo a 0,5 m. Os municípios envolvidos no estudo foram: Marechal Cândido Rondon (praia de Porto Mendes), Entre Rios do Oeste, Santa Helena (Balneário e Porto Internacional de Santa Helena), Itaipulândia (praia da Jacutinga), Santa Terezinha de Itaipu, Foz do Iguaçu (praia de Três Lagoas), São Miguel do Iguaçu (Balneário Ipiranga) e Missal. Itaipu é a hidrelétrica mais a jusante entre todas as demais existentes, ou em construção, na bacia do Rio Paraná, em território brasileiro. A área 
de drenagem do Rio Paraná até a barragem de Itaipu é de 820.000 $\mathrm{km}^{2}$, cerca de $25 \%$ de toda a área da bacia do Prata, abrangendo os municípios da costa oeste, de Foz do Iguaçu a Guaíra. O volume médio do reservatório é de 20 bilhões de $\mathrm{m}^{3}$, com profundidade média de $21,5 \mathrm{~m}$, tempo de residência da água de 40 dias e a velocidade média superficial de $0,6 \mathrm{~m} \mathrm{~s}^{-1}$. Soma-se um total de 66 ilhas, com uma área de proteção de 63.000 hectares ao longo de toda divisa das terras de Itaipu, no lado brasileiro, com cerca de $1.400 \mathrm{~km}$ de extensão. Foi plantada mata ciliar às margens do lago com mais de 1 milhão de mudas, formada por grupos de árvores nativas de rápido crescimento. ${ }^{9}$ A geologia regional é típica da Serra Geral, que ocupa grande parte do sudoeste e sul do Brasil e leste do Paraguai. Caracteriza-se por ser notadamente uniforme, de espessos derrames basálticos horizontais intercalados uniformemente por camadas de brecha, também conhecida por "Trap do Paraná". ${ }^{10}$

Nas amostragens e armazenagens de sedimento para avaliar os compostos inorgânicos foram utilizados coletores, colheres e embalagens plásticas. Para a determinação dos compostos orgânicos foram utilizados coletores de metal e a armazenagem foi realizada em recipientes de alumínio de $500 \mathrm{~mL}$. As amostragens foram realizadas utilizando-se protocolos limpos. ${ }^{11}$ Ambas as frações de amostras de sedimento foram mantidas em caixas de isopor com gelo e encaminhadas ao laboratório. O preparo das amostras de sedimentos para as análises químicas seguiu as etapas de secagem em temperatura ambiente, desagregação homogênea das partículas, maceração em almofariz, separação das partículas em malha de Teflon de $70 \mu \mathrm{m}$ e posterior armazenagem em frascos de vidro e embalagens plásticas sob refrigeração. Todo o material utilizado nas coletas e ensaios laboratoriais foi previamente descontaminado em banho de ácido nítrico $10 \% \mathrm{v} / \mathrm{v}$ por $24 \mathrm{~h}$. A água desionizada utilizada foi proveniente de um aparelho Human UP $900^{\circledR}$.

\section{Determinação de parâmetros físico-químicos e dos íons metálicos}

$\mathrm{O}$ fosfato total $\left(\mathrm{P}_{\mathrm{T}}\right)$ foi determinado em amostras de sedimento (aproximadamente $1,000 \mathrm{~g}$ ) utilizando-se $10 \mathrm{~mL}$ de solução extratora Mehlich-1 ( $\mathrm{H}_{2} \mathrm{SO}_{4}$ 0,025 mol L$\left.{ }^{-1} ; \mathrm{HCl} \mathrm{0,05} \mathrm{mol} \mathrm{L}{ }^{-1}\right)$. Após $12 \mathrm{~h}$ de repouso, adicionaram-se $5 \mathrm{~mL}$ de solução de molibdato de amônio 4,85 $\mathrm{mmol} \mathrm{L}^{-1}$ e uma pitada de ácido ascórbico em pó. A leitura foi realizada após $1 \mathrm{~h}$ de repouso em espectrofotômetro UV-Vis, Varian, Cary $50(660 \mathrm{~nm}) .{ }^{8}$ A quantificação de COT foi realizada pelo método da oxidação de matéria orgânica do sedimento (aproximadamente $1,000 \mathrm{~g})$ com $10 \mathrm{~mL}$ de solução de dicromato de potássio $\left(0,5 \mathrm{~mol} \mathrm{~L}^{-1}\right)$ em presença $20 \mathrm{~mL}$ de ácido sulfúrico concentrado. A titulação do excesso de dicromato na reação foi realizada com sulfato ferroso amoniacal $\left(0,25 \mathrm{~mol} \mathrm{~L}^{-1}\right)$ e ferroína $1 \% \mathrm{v} / \mathrm{v}$ como indicador. $\mathrm{O} \mathrm{pH}$ foi determinado pela estimativa da atividade dos íons $\mathrm{H}^{+}$na suspensão do sedimento, utilizando-se $\mathrm{CaCl}_{2} 10^{-2} \mathrm{~mol} \mathrm{~L}^{-1}$ para manter o equilíbrio iônico da solução. ${ }^{11} \mathrm{~A}$ análise granulométrica foi realizada através do método do densímetro, conhecido também como método do hidrômetro. ${ }^{12}$ Com ajuda de um densímetro foi possível relacionar as densidades com o tempo de leitura e com a temperatura, calculandose, com esses dados, a porcentagem das partículas nas frações argila, silte e areia. A determinação dos metais $\mathrm{Cd}, \mathrm{Cr}, \mathrm{Cu}, \mathrm{Fe}, \mathrm{Mn}, \mathrm{Ni}, \mathrm{Pb}$, $\mathrm{Al}$ e $\mathrm{Zn}$ foi realizada a partir da extração ácida das amostras com $\mathrm{HNO}_{3}$. Pesou-se aproximadamente $1,000 \mathrm{~g}$ de sedimento seco em béqueres de $250 \mathrm{~mL}$ e foram adicionados $10 \mathrm{~mL}$ de ácido nítrico concentrado. A solução permaneceu em repouso durante $12 \mathrm{~h}$. A seguir, as soluções foram aquecidas até $60^{\circ} \mathrm{C}$ por $2 \mathrm{~h}$, resfriadas à temperatura ambiente, adicionados $5 \mathrm{~mL}$ de peróxido de hidrogênio $30 \%(\mathrm{v} / \mathrm{v})$ e aquecidas a $60{ }^{\circ} \mathrm{C}$ por $30 \mathrm{~min}$. O produto da extração foi centrifugado e sofreu lavagens sucessivas com água desionizada.
O centrifugado foi avolumado a $50 \mathrm{~mL}$ e armazenado a $4{ }^{\circ} \mathrm{C}$ para a determinação da concentração dos metais. ${ }^{13,14}$ Todas as medidas dos metais foram efetuadas por espectroscopia de absorção atômica em chama (FAAS). As medidas de absorbância foram realizadas no modo integração de área, através do método da curva de calibração com os padrões dos metais avaliados. O LD dos metais foi considerado igual a três vezes o desvio estimado da curva analítica de calibração (Tabela 1S, material suplementar). A metodologia foi monitorada com material de referência certificado MRC-PACs 2 para verificar a porcentagem de metais extraída no processo.

\section{Análises das correlações entre os parâmetros avaliados}

Com o objetivo de se estudar possíveis relações entre os teores dos metais e os parâmetros físico-químicos encontrados nos sedimentos, foram calculadas as correlações, verificando-se a existência de uma possível relação linear entre dois dos valores quantitativos através do coeficiente $r$. Para se identificar grupos de elementos com comportamento similar e o comportamento geral dos dados, foi utilizada a técnica quimiométrica de classificação, a análise de componentes principais (ACP), avaliando-se o conjunto de dados obtidos e obtendo-se uma redução da dimensão dos mesmos, conservando a maior parte das informações estatísticas úteis presentes nos dados originais. As operações estatísticas foram realizadas através do programa Statistic versão 6.0.

\section{RESULTADOS E DISCUSSÃO}

\section{Parâmetros físico-químicos}

Os valores de $\mathrm{pH}$, nos nove pontos estudados, indicaram um caráter neutro a levemente ácido, sendo os valores mais baixos registrados nos pontos 5 (Praia de Foz do Iguaçu - Três Lagoas) e 8 (Praia de Itaipulândia - Jacutinga) (Tabela 1). A maior diferença entre os valores ocorreu entre os pontos 3 e 5 (2,97 unidades de $\mathrm{pH}$ ).

Tabela 1. Caracterização físico-química das amostras de sedimento superficial das praias do Lago de Itaipu

\begin{tabular}{lcccccc}
\hline $\begin{array}{l}\text { Pontos de } \\
\text { Coleta }\end{array}$ & $\mathrm{pH}$ & $\begin{array}{c}\mathrm{P}_{\mathrm{T}} \\
\left(\mathrm{mg} \mathrm{kg}^{-1}\right)\end{array}$ & $\begin{array}{c}\text { COT } \\
(\%)\end{array}$ & $\begin{array}{c}\text { Areia } \\
(\%)\end{array}$ & $\begin{array}{c}\text { Silte } \\
(\%)\end{array}$ & $\begin{array}{c}\text { Argila } \\
(\%)\end{array}$ \\
\hline 1 & 6,22 & 14,3 & 1,60 & 34 & 14 & 52 \\
2 & 6,18 & 20,6 & 1,51 & 86 & 2 & 12 \\
3 & 7,37 & 17,8 & 1,49 & 46 & 6 & 48 \\
4 & 6,55 & 55,7 & 1,07 & 42 & 16 & 42 \\
5 & 4,40 & 9,8 & 1,48 & 2 & 14 & 84 \\
6 & 6,60 & 1,3 & 1,54 & 44 & 10 & 46 \\
7 & 5,72 & 18,2 & 1,76 & 84 & 4 & 12 \\
8 & 5,00 & 10,6 & 1,71 & 70 & 2 & 28 \\
9 & 6,36 & 13,2 & 1,74 & 42 & 14 & 44 \\
\hline
\end{tabular}

Os sedimentos caracterizados mostram diferenças quanto ao teor de $\mathrm{P}_{\mathrm{T}}$ nos pontos em estudo. A maior concentração de $\mathrm{P}_{\mathrm{T}}$ no ponto 4 (Porto Internacional de Santa Helena) já era esperada, pois neste ocorrem derramamentos de adubos inorgânicos dos caminhões que transportam estes produtos entre o Brasil e o Paraguai quando sobem nas balsas. Este local pode ser classificado como um ambiente fortemente eutrofizado, devido à elevada presença de plantas aquáticas e com 43 vezes mais fosfato que o ponto 6 (Praia Santa Terezinha). Logo, a baixa concentração no ponto 6 , possivelmente, ocorre devido à originária fonte natural, como do intemperismo de rochas e decomposição de plantas. Já os pontos 1 e 2 recebem a influência de rios que deságuam próximos a esses locais e que a jusante recebem 
impactos de atividades antrópicas desenvolvidas às margens, principalmente agropecuária, justificando a maior concentração do $\mathrm{P}_{\mathrm{T}}$ quando comparado aos demais locais. Com exceção do ponto 4, as concentrações encontradas mostram um nível trófico semelhante aos apresentados na literatura para pontos localizados em áreas diferentes, mas no mesmo ambiente aquático. ${ }^{8}$

O teor de COT oscilou em torno de $1,50 \%$, esses valores revelam sedimentos de natureza mineral, com moderado conteúdo de matéria orgânica, pois apresentam menos de $10 \%$ de matéria orgânica em peso seco $\left(\% \mathrm{MO}=\% \mathrm{C}\right.$ x 1,725). ${ }^{15} \mathrm{O}$ fato de os valores de COT não serem muito altos é justificado pelos locais de coleta (praias artificiais) onde um grande conteúdo mineral foi adicionado (areia).

Com relação à influência da matéria orgânica na retenção de metais, cabe ressaltar a dificuldade de comparação entre os vários trabalhos existentes na literatura. Isso porque, como se sabe, a matéria orgânica apresenta diversos grupamentos funcionais reativos, responsáveis pela retenção de metais, tais como aminas; iminos; carboxilas; fenólicos; alcoólicos; carbonilas e sulfidrilas. A matéria orgânica que apresenta em sua estrutura a predominância de grupos carboxílicos certamente será diferente de outra em que prevaleçam grupos nitrogenados. Assim, o ideal, antes de se proceder aos experimentos de adsorção, seria caracterizar a matéria orgânica presente. ${ }^{15}$ Entretanto, devido aos baixos teores de COT, é esperada uma elevada disponibilidade dos metais para o ambiente aquático, o que pode facilitar um processo de biomagnificação. ${ }^{16}$ As amostras foram classificadas texturalmente com relação à porcentagem de cada fração encontrada com a análise granulométrica. Os pontos 1, 3, 4, 6 e 9 possuem classificação textural argilosa; 5 foi classificado como muito argiloso; os pontos 2 e 7 como arenosos e 8 como médio arenoso.

\section{Extração e determinação dos íons metálicos}

Foi utilizado o MRC-PACs 2 com o intuito de se conhecer a concentração recuperada de cada metal estudado no processo de extração da fração ambientalmente disponível. O processo da pseudo-extração ácida resultou na recuperação das seguintes porcentagens de metais na amostra de sedimento do MRC: $\mathrm{Al}(21,9 \%), \mathrm{Cd}(97,2 \%), \mathrm{Cr}(36,7 \%)$, $\mathrm{Cu}(93,5 \%), \mathrm{Fe}(71,6 \%), \mathrm{Mn}(48,6 \%), \mathrm{Ni}(15,4 \%), \mathrm{Pb}(110,0 \%)$ e $\mathrm{Zn}$ $(53,3 \%)$. Pode-se perceber através desta avaliação que a disponibilidade dos metais para o meio através da pseudo-extração diminuiu na ordem $\mathrm{Pb}>\mathrm{Cd}>\mathrm{Cu}>\mathrm{Fe}>\mathrm{Zn}>\mathrm{Mn}>\mathrm{Cr}>\mathrm{Al}>\mathrm{Ni}$ (Tabela $2 \mathrm{~S}$, material suplementar). Os resultados obtidos, com base na recuperação do MRC, com as determinações dos metais nas amostras coletadas em cada ponto de amostragem encontram-se na Tabela 2. Para melhor visualização da concentração dos metais nos pontos de amostragem do Lago de Itaipu, foi realizada uma distribuição espacial dos metais estudados, Figura 1A e B.

A legislação ambiental brasileira ainda não dispõe de critérios de qualidade para sedimento e também não existem dados de Nível de Base Natural (NBN ou background) para os parâmetros
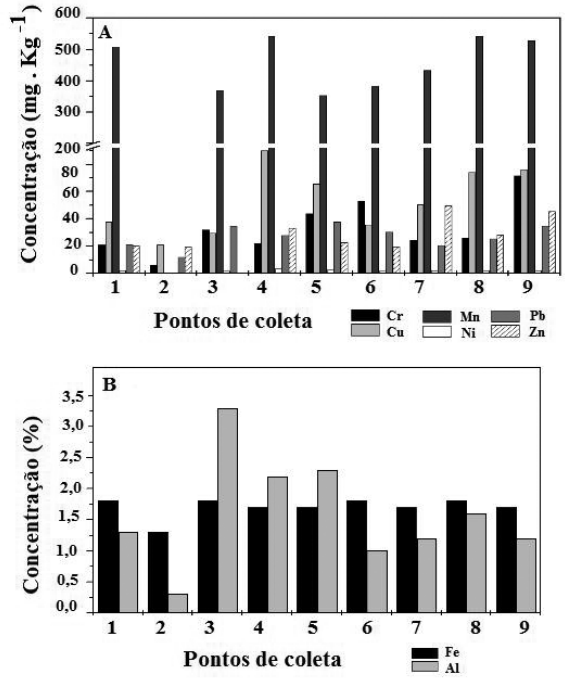

Figura 1. Distribuição espacial dos teores de metais nos sedimentos do Lago de Itaipu (A) em $\mathrm{mg} \mathrm{kg}^{-1}$ e (B) em \% para Al e Fe

determinados nos sedimentos das regiões em estudo. Sendo assim, para efeito de comparação e para uma aproximação sobre as consequências dos teores de metais nos sedimentos, foram utilizados os critérios estabelecidos na literatura, representadas por agências ambientais internacionais, como a NOAA - National Oceanic and Atmosferic and Administration (Tabela 3S, material suplementar). ${ }^{17}$ As concentrações dos metais, com base na NOAA, revelaram que os teores de $\mathrm{Fe}$ foram sempre inferiores ao SEL (efeito de grau severo), 4\%, em todos os pontos avaliados. Para o metal $\mathrm{Cu}$, os pontos $1,4,5,6,7,8$ e 9 apresentaram concentrações superiores ao TEL (valores abaixo dos quais efeitos adversos são raramente esperados), $35,7 \mathrm{mg} \mathrm{kg}^{-1}$, e inferiores ao PEL (nível de efeitos prováveis), $197,0 \mathrm{mg} \mathrm{kg}^{-1}$. Em relação ao elemento Mn, todos os pontos apresentaram concentrações menores que o SEL (1.100,00 mg kg-1). Para o Al não existem valores classificados pela NOAA para PEL, TEL e SEL. Para o Zn, os pontos apresentaram teores inferiores ao TEL, 123,0 $\mathrm{mg} \mathrm{kg}^{-1}$. O Cd apresentou concentrações abaixo do LD do método analítico empregado em todas as amostras. Observou-se que o valor de $\mathrm{Cr}$ se encontra abaixo do TEL em vários pontos estudados, exceto nos pontos 5 , 6 e 9 onde está acima do TEL (37,3 $\left.\mathrm{mg} \mathrm{kg}^{-1}\right)$. Para o $\mathrm{Ni}$, segundo os valores adotados pela NOAA, nenhum ponto de coleta excedeu o valor do nível TEL (18,0 mg kg-1). O metal $\mathrm{Pb}$ foi encontrado em valores inferiores ao TEL $\left(35,0 \mathrm{mg} \mathrm{kg}^{-1}\right)$ e superiores apenas para os pontos 5 (Praia de Foz do Iguaçu) e 9 (Praia de Missal), no entanto, abaixo de PEL (91,3 $\left.\mathrm{mg} \mathrm{kg}^{-1}\right)$.

Tabela 2. Resultados das concentrações dos metais (em mg kg${ }^{-1}$, exceto $\mathrm{Al}$ e Fe em \%) nos sedimentos do Lago de Itaipu

\begin{tabular}{|c|c|c|c|c|c|c|c|c|c|}
\hline Pontos & $\mathrm{Al}$ & $\mathrm{Cd}$ & $\mathrm{Cr}$ & $\mathrm{Cu}$ & $\mathrm{Fe}$ & $\mathrm{Mn}$ & $\mathrm{Ni}$ & $\mathrm{Pb}$ & $\mathrm{Zn}$ \\
\hline 1 & $1,3 \pm 0,0$ & $<\mathrm{LD}$ & $21,3 \pm 0,6$ & $37,8 \pm 3,4$ & $1,8 \pm 0,02$ & $526,0 \pm 34,0$ & $2,2 \pm 0,2$ & $21,0 \pm 0,7$ & $20,2 \pm 1,6$ \\
\hline 2 & $0,3 \pm 0,0$ & $<\mathrm{LD}$ & $6,4 \pm 0,3$ & $21,0 \pm 1,7$ & $1,3 \pm 0,01$ & $182,0 \pm 11,0$ & $<\mathrm{LD}$ & $12,0 \pm 0,6$ & $19,7 \pm 1,1$ \\
\hline 3 & $3,3 \pm 1,8$ & $<\mathrm{LD}$ & $32,2 \pm 2,7$ & $29,9 \pm 5,3$ & $1,8 \pm 0,01$ & $371,0 \pm 1,0$ & $2,0 \pm 0,2$ & $34,5 \pm 0,5$ & $108 \pm 1,0$ \\
\hline 4 & $2,2 \pm 0,3$ & $<\mathrm{LD}$ & $22,5 \pm 0,2$ & $90,0 \pm 5,2$ & $1,7 \pm 0,03$ & $543,0 \pm 5,0$ & $3,6 \pm 0,6$ & $28,2 \pm 2,3$ & $33,4 \pm 2,2$ \\
\hline 5 & $2,3 \pm 0,2$ & $<\mathrm{LD}$ & $43,8 \pm 1,6$ & $65,9 \pm 2,9$ & $1,7 \pm 0,02$ & $355,0 \pm 2,0$ & $2,7 \pm 0,2$ & $38,1 \pm 3,6$ & $22,9 \pm 1,6$ \\
\hline 6 & $1,0 \pm 0,2$ & $<\mathrm{LD}$ & $53,4 \pm 4,8$ & $35,8 \pm 2,9$ & $1,8 \pm 0,01$ & $383,0 \pm 8,0$ & $1,9 \pm 0,2$ & $30,7 \pm 3,2$ & $19,4 \pm 1,0$ \\
\hline 7 & $1,2 \pm 0,1$ & $<\mathrm{LD}$ & $24,3 \pm 0,5$ & $50,4 \pm 3,2$ & $1,7 \pm 0,01$ & $434,0 \pm 2,0$ & $2,2 \pm 0,2$ & $20,7 \pm 1,8$ & $49,7 \pm 3,5$ \\
\hline 8 & $1,6 \pm 0,1$ & $<\mathrm{LD}$ & $26,2 \pm 1,7$ & $74,1 \pm 3,4$ & $1,8 \pm 0,02$ & $543,0 \pm 7,0$ & $2,4 \pm 0,0$ & $25,5 \pm 3,5$ & $28,7 \pm 1,1$ \\
\hline 9 & $1,2 \pm 0,1$ & $<\mathrm{LD}$ & $71,9 \pm 1,6$ & $76,1 \pm 1,3$ & $1,7 \pm 0,01$ & $529,0 \pm 11,0$ & $2,4 \pm 0,0$ & $35,0 \pm 4,5$ & $45,7 \pm 0,2$ \\
\hline
\end{tabular}


Determinou-se o fator de contaminação (FC) para alguns metais, no intuito de identificar as estações de coleta que se afastam das condições naturais em função das atividades antropogênicas realizadas ao longo do Lago de Itaipu. Para essa classificação, optou-se por utilizar como NBN os valores médios globais da Agência de Proteção Ambiental - EPA apud Rodrigues, ${ }^{18}$ e de Turekian e Wedephol. ${ }^{19} \mathrm{O}$ FC dos sedimentos foi calculado através da razão entre a concentração do elemento no sedimento superficial e a concentração do seu NBN ou background. ${ }^{7,8}$ Essa metodologia permite verificar em que ordem de grandeza o nível natural dos metais foi excedido, indicando o enriquecimento ou, caso contrário, o empobrecimento do elemento no sedimento. ${ }^{20}$ Neste trabalho foi utilizada a classificação de Hakanson que propõe que valores de FC menores que 1 indicam baixa contaminação, valores entre 1 e 3 contaminação moderada, valores entre 3 e 6 contaminação considerável e valores maiores que 6 alta contaminação. ${ }^{7}$ A Figura 2 representa os histogramas de distribuição dos FC calculados para os pontos de coleta do Lago de Itaipu $(\mathrm{Cr}, \mathrm{Cu}, \mathrm{Ni}, \mathrm{Pb}$ e $\mathrm{Zn})$ segundo a EPA apud Rodrigues, ${ }^{18}$ e Turekian e Wedephol ${ }^{19}$ (Tabelas 4S e 5S, material suplementar).

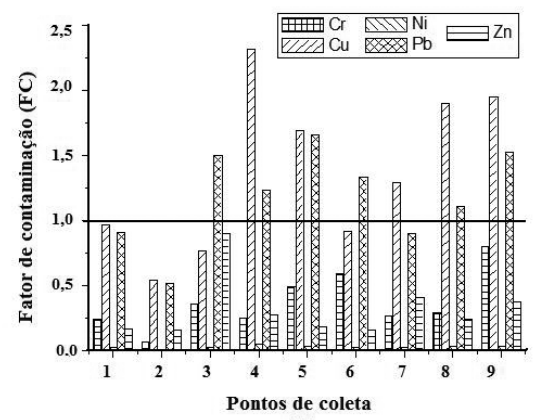

(A)

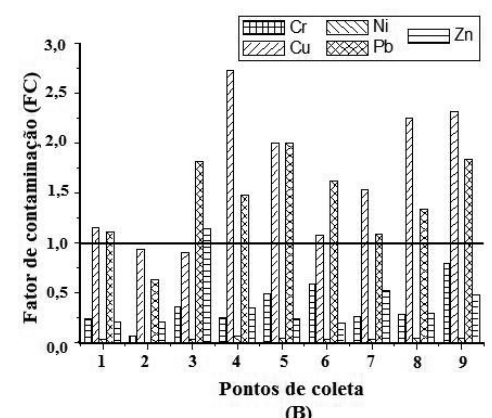

(B)

Figura 2. Histogramas de distribuição dos fatores de contaminação calculados para os pontos de coleta do Lago de Itaipu ( $\mathrm{Cr}, \mathrm{Cu}, \mathrm{Ni}, \mathrm{Pb}$ e $\mathrm{Zn}$ ) segundo (A) NBN adaptado de EPA (apud Rodrigues) ${ }^{18}$ e (B) Turekian e Wedephol ${ }^{19}$

De acordo com os histogramas, nos nove pontos estudados na região, os dois elementos que apresentaram maior afastamento da condição "natural", em termos de incremento, foram $\mathrm{Cu}$ e $\mathrm{Pb}$. Os metais $\mathrm{Cu}$ e $\mathrm{Pb}$, sob condições oxidantes e em meio ácido, são solúveis e possuem uma boa mobilidade nos sistemas aquáticos. Com diminuição do $\mathrm{pH}$, suas concentrações na coluna d'água tendem a aumentar, logo, o pH observado gera um meio pouco ácido, possibilitando a adsorção pelos óxidos e hidróxidos de manganês (principalmente $\mathrm{Cu}$ ) e a formação de carbonatos que são compostos de baixa solubilidade, facilitando a deposição no sedimento. Com isso, a concentração acentuada do metal $\mathrm{Cu}$ nos sedimentos em estudo pode ser explicada baseada nessa característica, já que o elemento Mn é encontrado em teor bem significativo..$^{10} \mathrm{O}$ maior incremento em
$\mathrm{Cu}$ e $\mathrm{Pb}$ foi observado, principalmente, onde há evidências de lançamento de cargas orgânicas e efluentes domésticos. Estes elementos se acumulam na fração fina dos sedimentos de fundo e dão indícios de poluição antropogênica. Podem-se citar como fontes antropogênicas na região do Lago de Itaipu os usos múltiplos instalados na margem brasileira do Reservatório, como praias artificiais, corredores usados pelo gado para tomar água, pontos de captação de água para consumo humano e irrigação de culturas agrícolas, portos de descarga de areia e produtos agrícolas, portos de travessia, pontos de pesca, criadores de peixes em tanques-rede e bases náuticas. No entanto, o uso e a ocupação do solo já ocorriam mesmo antes da construção da represa, pois a área inundada era explorada por atividades agropecuárias bem como por rodovias pré-existentes.

\section{Correlações entre os teores de metais e parâmetros físico- químicos}

Objetivando revelar associações que dão informações sobre processos geoquímicos que controlam ou influenciam na distribuição dos elementos nos sedimentos, foi calculado o coeficiente de correlação entre os parâmetros físico-químicos analisados. Destacaram-se correlações positivas entre $\mathrm{Cu}-\mathrm{Mn}(\mathrm{r}=0,710), \mathrm{Fe}-\mathrm{Mn}(\mathrm{r}=0,743), \mathrm{Cu}-\mathrm{Ni}$ $(\mathrm{r}=0,853), \mathrm{Cr}-\mathrm{Pb}(\mathrm{r}=0,771), \mathrm{Fe}-\mathrm{Pb}(\mathrm{r}=0,739)$ e $\mathrm{Ni}-\mathrm{P}_{\mathrm{T}}(\mathrm{r}=0,834)$ e correlações negativas entre $\mathrm{P}_{\mathrm{T}}$-COT. Também foi possível notar a baixa correlação entre Cr-Zn, Mn-Zn, Cr-pH, Mn-pH, Pb-pH, Zn-P, Fe-COT, Fe-COT, Mn-COT e Zn-COT. A partir das correlações com os metais estudados pode-se perceber que $\mathrm{pH}$ e COT não são determinantes para explicar as suas concentrações (Tabela $6 \mathrm{~S}$, material suplementar).

A análise de componentes principais gerou novas variáveis, combinações lineares das variáveis originais, que foram usadas para analisar os dados através de projeção geométrica. O modelo estatístico usado permitiu discriminar os pontos de coleta, bem como identificar de forma rápida e eficaz as relações existentes entre os dados. Como pode ser observado na Figura 3, a análise estatística mostrou que a soma das duas primeiras componentes principais explicou $63,40 \%$ da variabilidade dos dados, sendo a componente principal 1 com maior contribuição (fator 44,28\%) das variáveis areia, $\mathrm{Ni}$, silte, $\mathrm{Cu}, \mathrm{Al}$, argila, $\mathrm{Pb}, \mathrm{Fe}$ e $\mathrm{Cr}$. A segunda componente principal com menor contribuição (fator 19,12\%) apenas nas variáveis $\mathrm{P}_{\mathrm{T}}$, COT e $\mathrm{Zn}$. A variável $\mathrm{pH}$ não foi incluída nesta análise, devido á pequena variação dos valores encontrados das amostras de sedimentos. Posicionando-se os resultados das amostras em um espaço bidimensional observa-se que os pontos 2 e 4 estão bastante afastados do agrupamento principal. Analisando-se os resultados obtidos para essas amostras, verifica-se que no ponto 2 o sedimento apresentou um nível de contaminação abaixo dos outros pontos de coleta para todos os metais. Entretanto, o ponto 4 se diferenciou do grupo principal devido às maiores concentrações dos metais $\mathrm{Cu}$ e Ni, com este último contribuindo significativamente para a componente principal 1. O COT foi encontrado nas amostras de sedimento em pequena quantidade, mas, através do gráfico de loadings, observouse boa correlação com os parâmetros $\mathrm{P}_{\mathrm{T}}, \mathrm{Cu}$ e Al. Outros trabalhos também utilizaram a ACP como ferramenta de análise de dados e citaram a importância da matéria orgânica (MO), aqui adotado o carbono orgânico total (COT x 1,725) como fase complexadora de metais-traço. ${ }^{20,21} \mathrm{~A}$ maioria dos metais avaliados apresentou-se no mesmo quadrante em relação à argila e silte, representando a maior afinidade pelas partículas menores dos sedimentos.

\section{CONCLUSÕES}

Este trabalho mostra que os sedimentos das praias artificiais do Lago de Itaipu alcançaram níveis de concentração para alguns metais 

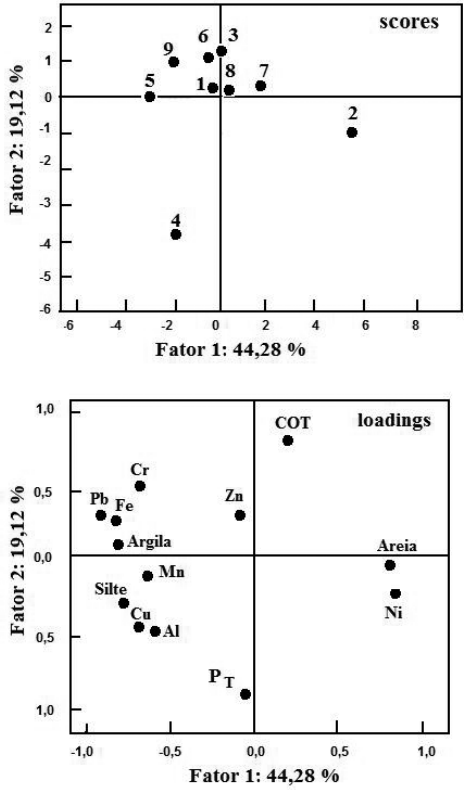

Figura 3. Projeção dos scores e loadings das amostras de sedimento (CP1 $x$ CP2) para os pontos estudados no Lago de Itaipu

que podem afetar o equilíbrio do ecossistema, segundo os critérios internacionais de classificação de sedimentos adotados pela NOAA. $\mathrm{O}$ fator de contaminação calculado apresentou incrementos em $\mathrm{Cu}$ e $\mathrm{Pb}$ nos sedimentos, indicando moderada contaminação, segundo a classificação de Hakanson. Quanto aos parâmetros físico-químicos, observa-se pH neutro a levemente ácido. As concentrações de $\mathrm{P}_{\mathrm{T}}$ levam a um baixo nível trófico. Os teores de COT revelam sedimentos de natureza mineral. Com relação à granulometria dos sedimentos, foi possível notar o predomínio da fração fina, argila.

A metodologia utilizada na extração dos metais foi monitorada com MRC no intuito de se conhecer a recuperação de cada metal no processo de pseudodigestão e compreender melhor a sua biodisponibilidade no ecossistema aquático. A concentração recuperada para o elemento $\mathrm{Ni}$ $(15,4 \%)$ foi muito baixa, mostrando que o mesmo está fracamente disponível para a biota, já para o $\mathrm{Pb}(110 \%)$, apresenta maior mobilidade em relação aos demais metais avaliados no sedimento superficial.

Os resultados analíticos apresentados, somados aos já existentes, podem contribuir para um futuro estabelecimento, pela legislação ambiental brasileira, de critérios nacionais para avaliar a qualidade de sedimentos. Também podem contribuir para alertar sobre a exploração consciente do Lago de Itaipu e das praias artificiais construídas ao longo dele. $\mathrm{O}$ uso das ferramentas estatísticas foi importante na avaliação da contaminação por metais, destacando a influência das variáveis sobre os pontos estudados.

\section{MATERIAL SUPLEMENTAR}

A Fígura 1S apresenta a localização do reservatório da Usina Hidrelétrica de Itaipu no Rio Paraná, fronteira do Brasil e do Pa- raguai, onde as amostras de sedimento foram coletadas. A Tabela 1S apresenta os parâmetros experimentais utilizados nas medidas dos metais por FAAS. A Tabela $2 \mathrm{~S}$ apresenta a concentração do MRC-PACs 2 e a concentração extraída após a pseudoextração. A Tabela 3S apresenta os valores guias de qualidade de sedimento de metais-traço adotados pela NOAA. As Tabelas $4 \mathrm{~S}$ e $5 \mathrm{~S}$ apresentam o Nível de Base Natural de alguns elementos e os cálculos de FC realizados para os sedimentos avaliados, respectivamente. A Tabela $6 \mathrm{~S}$ apresenta a matriz de correlação entre as variáveis dos sedimentos. Está disponível em http://quimicanova.sbq.org.br, na forma de aquivo PDF, com acesso livre.

\section{REFERÊNCIAS}

1. Jardim, G. M.; Armas, E. D.; Monteiro, R. T. R.; Braz. J. Biol. 2008, 68, 51.

2. Cotta, J. A. O.; Rezende, M. O. O.; Piovani, M. R.; Quim. Nova 2006, $29,40$.

3. Pereira, C. D.; Quináia, S. P.; Ambiência 2007, 3, 27.

4. Seiler, H. G., Sigel, H.; Handbook on Toxicity of Inorganic Compounds, Marcel Dekker, Inc: New York, 1988.

5. Vandecasteele, C.; Block, C .B.; Modern Methods for Trace Element Determination, John Wiley \& Sons: New York, 1997.

6. Baird, C.; Química Ambiental, 2a ed., Bookman: Porto Alegre, 2002.

7. Hakanson, L.; Water Res. 1980, 14, 975.

8. Pletsch, A. L.; Dissertação de Mestrado, Universidade Estadual de Londrina, Brasil, 2002.

9. http://www.itaipu.gov.br, acessada em Setembro 2008.

10. Pavan, M. A.; Bloch, M. F.; Zempulski, H. C.; Miyazawa, M.; Zocoler, D. C.; Manual de Análise Química de Solo e Controle de Qualidade, Instituto Agronômico do Paraná: Londrina, 1992.

11. Hansen, H. P.; Koroleff, F. Em Methods of Seawater Analysis; Grasshoff, K.; Kremling, K.; Ehrhardt, M., eds.; $2^{\text {nd }}$ ed., Wiley: New York, 1999 , cap. 10.

12. EMBRAPA; Manual de métodos de análise de solos, 2ª ed., EMBRAPA: Rio de Janeiro, 1997.

13. Pletsch, A. L.; Tese de Doutorado, Universidade Estadual da Bahia, Brasil, 2006.

14. Rheinheimer, D. S.; Campos, B. C.; Giacomini, S. J.; Conceição, P. C.; Bortoluzzi, E. C.; Rev. Bras. Ciênc. Solo 2008, 32, 435.

15. Pierangeli, M. A. P.; Guilherme, L. R. G.; Curi, N.; Silva, M. L. N.; Lima, J. M.; Costa, E. T. S.; Rev. Bras. Ciênc. Solo 2005, 29, 523.

16. Sujatha, C. H.; Aneeshkumar, N.; Renjith, K. R.; Current Sci. 2008, 95, 382 .

17. http://response.restoration.noaa.gov/book_shelf/122_NEW-SQuiRTs. pdf, acessada em Setembro 2009.

18. Rodrigues, M. L. K.; Dissertação de Mestrado, Universidade Federal do Rio Grande do Sul, Brasil, 1997.

19. Turekian, K. K; Wedepohl, K. H.; Geol. Soc. Am. Bull. 1961, 72, 175.

20. Silva, W. L.; Machado, W.; Matos, R. H. R.; J. Braz. Chem. Soc. 2008, $19,1490$.

21. Clarke, K. M.; Warwick, R. M.; Change in marine communities: an approach to statistical analysis and interpretation, Plymouth Marine Laboratory: Plymouth, 1994. 


\section{AVALIAÇÃO DA CONTAMINAÇÃO DE METAIS EM SEDIMENTOS SUPERFICIAIS DAS PRAIAS DO LAGO} DE ITAIPU

\section{Andressa Belo e Sueli Pércio Quináia*}

Departamento de Química, Universidade Estadual do Centro-Oeste, Rua Camargo Varela de Sá, 3, 85040-080 Guarapuava - PR, Brasil Adelmo Lowe Pletsch

Departamento de Ciências Biológicas e Tecnologia, Universidade Estadual do Norte do Paraná, BR 369, km 54, 86360-000 Bandeirantes - PR, Brasil

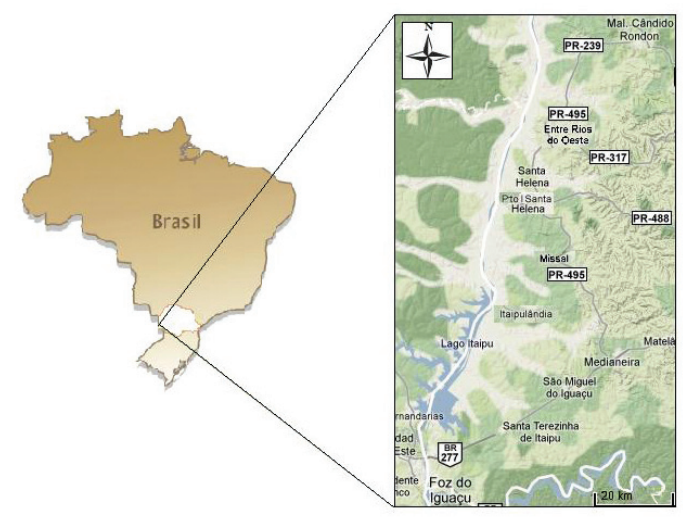

Figura 1S. Localização do reservatório da Usina Hidrelétrica de Itaipu no Rio Paraná, fronteira do Brasil e do Paraguai, onde as amostras de sedimento foram coletadas

Tabela 1S. Parâmetros experimentais das medidas de metais por FAAS

\begin{tabular}{lcccccc}
\hline \hline Metais & $\begin{array}{c}\lambda \\
(\mathrm{nm})\end{array}$ & $\begin{array}{c}\text { i lâmpada } \\
(\mathrm{mA})\end{array}$ & $\begin{array}{c}\text { Fenda } \\
(\mathrm{nm})\end{array}$ & Chama & $\mathrm{r}$ & $\begin{array}{c}\mathrm{LD}^{-1} \\
\left(\mathrm{mg} \mathrm{L}^{-}\right)\end{array}$ \\
\hline $\mathrm{Al}$ & 309,3 & 10 & 0,5 & $\mathrm{~N}_{2} \mathrm{O}_{\mathrm{C}} \mathrm{H}_{2}$ & 1,0000 & 0,173 \\
$\mathrm{Cd}$ & 228,8 & 4 & 0,5 & $\mathrm{Ar} / \mathrm{C}_{2} \mathrm{H}_{2}$ & 0,9992 & 0,030 \\
$\mathrm{Cr}$ & 357,9 & 7 & 0,2 & $\mathrm{Ar} / \mathrm{C}_{2} \mathrm{H}_{2}$ & 0,9994 & 0,052 \\
$\mathrm{Cu}$ & 324,7 & 4 & 0,5 & $\mathrm{Ar} / \mathrm{C}_{2} \mathrm{H}_{2}$ & 1,0000 & 0,145 \\
$\mathrm{Fe}$ & 248,3 & 5 & 0,2 & $\mathrm{Ar} / \mathrm{C}_{2} \mathrm{H}_{2}$ & 1,0000 & 0,064 \\
$\mathrm{Mn}$ & 279,5 & 5 & 0,2 & $\mathrm{Ar} / \mathrm{C}_{2} \mathrm{H}_{2}$ & 0,9999 & 0,093 \\
$\mathrm{Ni}$ & 232,0 & 4 & 0,2 & $\mathrm{Ar} / \mathrm{C}_{2} \mathrm{H}_{2}$ & 0,9986 & 0,035 \\
$\mathrm{~Pb}$ & 217,0 & 5 & 1,0 & $\mathrm{Ar} / \mathrm{C}_{2} \mathrm{H}_{2}$ & 0,9999 & 0,287 \\
$\mathrm{Zn}$ & 213,9 & 5 & 1,0 & $\mathrm{Ar} / \mathrm{C}_{2} \mathrm{H}_{2}$ & 0,9994 & 0,180 \\
\hline
\end{tabular}

LD = Limite de detecção
Tabela 2S. Concentração dos metais no MRC-PACs 2 e concentração extraída no processo de pseudoextração ácida. $\mathrm{Cd}, \mathrm{Cr}, \mathrm{Cu}, \mathrm{Mn}, \mathrm{Ni}, \mathrm{Pb}$ e $\mathrm{Zn}$ (mg . $\mathrm{Kg}-1)$ e $\mathrm{Al}$ e $\mathrm{Fe}(\%)$

\begin{tabular}{lccc}
\hline Metal & Concentração MRC & Concentração extraída & \% extraída \\
\hline $\mathrm{Al}$ & $6,62 \pm 0,32$ & $1,45 \pm 0,02$ & 21,9 \\
$\mathrm{Cd}$ & $2,11 \pm 0,15$ & $2,05 \pm 0,15$ & 97,2 \\
$\mathrm{Cr}$ & $90,70 \pm 4,60$ & $33,30 \pm 0,60$ & 36,7 \\
$\mathrm{Cu}$ & $310,00 \pm 12,00$ & $290,00 \pm 3,00$ & 93,5 \\
$\mathrm{Fe}$ & $4,09 \pm 0,06$ & $2,93 \pm 0,04$ & 71,6 \\
$\mathrm{Mn}$ & $440,00 \pm 19,00$ & $214,00 \pm 4,00$ & 48,6 \\
$\mathrm{Ni}$ & $39,50 \pm 2,30$ & $6,10 \pm 0,40$ & 15,4 \\
$\mathrm{~Pb}$ & $183,00 \pm 12,00$ & $205,00 \pm 2,00$ & 112,0 \\
$\mathrm{Zn}$ & $364,00 \pm 23,00$ & $194,00 \pm 7,00$ & 53,3 \\
\hline
\end{tabular}

*e-mail: spquinaia@gmail.com 
Tabela 3S. Valores guias de qualidade de sedimento de metais-traço em sedimentos adotados pela NOAA* $\left(\mathrm{mg} \mathrm{kg}^{-1}\right.$, base seca)

\begin{tabular}{lccccccccc}
\hline Indicador & $\mathrm{Al}$ & $\mathrm{Cd}$ & $\mathrm{Cr}$ & $\mathrm{Cu}$ & $\mathrm{Fe}$ & $\mathrm{Mn}$ & $\mathrm{Ni}$ & $\mathrm{Pb}$ & $\mathrm{Zn}$ \\
\hline TEL & -- & 0,59 & 37,3 & 35,7 & -- & -- & 18 & 35 & 123 \\
PEL & -- & 3,5 & 90 & 197 & -- & -- & 36 & 91,3 & 315 \\
SEL & -- & 10 & 110 & 110 & $4 \%$ & 1100 & 75 & 250 & 820 \\
\hline
\end{tabular}

* Diretrizes de qualidade para priorização de áreas a serem estudadas detalhadamente e priorização de contaminantes químicos. TEL - valor abaixo dos quais efeitos adversos são raramente esperados; PEL - nível de efeitos prováveis; SEL - nível de efeitos severos.

Tabela 4S. Nível de Base Natural de alguns elementos estudados (em mg kg-1)

\begin{tabular}{llllll}
\hline NBN & $\mathrm{Cr}$ & $\mathrm{Cu}$ & $\mathrm{Ni}$ & $\mathrm{Pb}$ & $\mathrm{Zn}$ \\
\hline EPA apud Rodrigues $^{18}$ & 90 & 39 & 68 & 23 & 120 \\
Turekian e Wedephol $^{19}$ & 90 & 33 & 52 & 19 & 95 \\
\hline
\end{tabular}

Tabela 5S. Fator de contaminação para os metais $\mathrm{Cr}, \mathrm{Cu}, \mathrm{Ni}, \mathrm{Pb}$ e $\mathrm{Zn}$ nos sedimentos do Lago de Itaipu utilizando como NBN os valores médios da EPA (apud Rodrigues) ${ }^{18}$ e Turekian e Wedephol ${ }^{19}$

\begin{tabular}{lcccccccccc}
\hline & \multicolumn{2}{c}{$\mathrm{Cr}$} & \multicolumn{2}{c}{$\mathrm{Cu}$} & \multicolumn{2}{c}{$\mathrm{Ni}$} & \multicolumn{2}{c}{$\mathrm{Pb}$} & \multicolumn{2}{c}{$\mathrm{Zn}$} \\
Pontos & EPA & TW & EPA & TW & EPA & TW & EPA & TW & EPA & TW \\
\hline 1 & 0,24 & 0,24 & 0,97 & 1,15 & 0,03 & 0,04 & 0,91 & 1,11 & 0,17 & 0,21 \\
2 & 0,07 & 0,07 & 0,54 & 0,94 & - & - & 0,52 & 0,63 & 0,16 & 0,21 \\
3 & 0,36 & 0,36 & 0,77 & 0,91 & 0,03 & 0,04 & 1,50 & 1,82 & 0,90 & 1,14 \\
4 & 0,25 & 0,25 & 2,31 & 2,73 & 0,05 & 0,07 & 1,23 & 1,48 & 0,28 & 0,35 \\
5 & 0,49 & 0,49 & 1,69 & 2,00 & 0,04 & 0,05 & 1,66 & 2,00 & 0,19 & 0,24 \\
6 & 0,59 & 0,59 & 0,92 & 1,08 & 0,03 & 0,04 & 1,33 & 1,62 & 0,16 & 0,20 \\
7 & 0,27 & 0,27 & 1,29 & 1,53 & 0,03 & 0,04 & 0,90 & 1,09 & 0,41 & 0,52 \\
8 & 0,29 & 0,29 & 1,90 & 2,25 & 0,04 & 0,05 & 1,11 & 1,34 & 0,24 & 0,30 \\
9 & 0,80 & 0,80 & 1,95 & 2,31 & 0,04 & 0,05 & 1,52 & 1,84 & 0,38 & 0,48 \\
\hline
\end{tabular}

Tabela 6S. Matriz de correlação entre as variáveis dos sedimentos para o Lago de Itaipu

\begin{tabular}{|c|c|c|c|c|c|c|c|c|c|c|c|}
\hline & $\mathrm{Al}$ & $\mathrm{Cr}$ & $\mathrm{Cu}$ & $\mathrm{Fe}$ & $\mathrm{Mn}$ & $\mathrm{Ni}$ & $\mathrm{Pb}$ & $\mathrm{Zn}$ & $\mathrm{pH}$ & $\mathrm{P}_{\mathrm{T}}$ & COT \\
\hline$\overline{\mathrm{Al}}$ & 1 "'," & 0,100 & $0,228^{\prime}$ & $0,617 "$ & 0,233 & 0,206 & $0,670 " ”$ & $0,698 "$ & $0,150^{\prime}$ & 0,240 & $-0,375$ \\
\hline $\mathrm{Cr}$ & & 1"'”' & $0,322^{\prime}$ & $0,529 ”$ & 0,293 & $-0,206$ & 0,771 "' & 0,089 & 0,026 & $-0,409$ '" & $0,276 ”$ \\
\hline $\mathrm{Cu}$ & & & $1 ", "$ & $0,463 "$ & $0,710 "$ & $0,853 "$, & $0,429 "$ & $-0,157$ & $-0,371$, & $0,439 "$ & $-0,233$ \\
\hline $\mathrm{Fe}$ & & & & $1 ", "$, & 0,743 "” & $-0,141$ & $0,739 "$ & 0,267 & $-0,026^{\prime}$ & $-0,139^{\prime}$ & 0,037 \\
\hline $\mathrm{Mn}$ & & & & & $1 ", "$ & $0,437 "$ & $0,299 "$ & $-0,001$ & $-0,033$, & $0,227 "$ & $0,036^{\prime}$ \\
\hline $\mathrm{Ni}$ & & & & & & $1 ", "$, & 0,100 & $-0,254 ”$ & $-0,204^{\prime}$ & 0,834 "”, & $-0,718 "$, \\
\hline $\mathrm{Pb}$ & & & & & & & $1, ", "$ & 0,350 & $-0,04$ & $-0,150$ & $-0,112$ \\
\hline $\mathrm{Zn}$ & & & & & & & & $1 ", "$ & $0,532 "$ & 0,08 & $0,032^{\prime}$ \\
\hline $\mathrm{pH}$ & & & & & & & & & $1 ", "$, & $0,264^{\prime}$ & $-0,528$ \\
\hline $\mathrm{P}_{\mathrm{T}}$ & & & & & & & & & & $1, "$, & $-0,77$ \\
\hline COT & & & & & & & & & & & $1 ", "$, \\
\hline
\end{tabular}

"'” $\mathrm{P}<0,0001$ "”' $\mathrm{P}<0,01$ "” $\mathrm{P}<0,10$ " $\mathrm{P}<0,25$ 'P $<0,50 \mathrm{P}<0,75$ 